\title{
Overexpression of fascin-1 in advanced colorectal adenocarcinoma: Tissue microarray analysis of immunostaining scores with clinicopathological parameters
}

\author{
Wen-Chiuan Tsai ${ }^{\mathrm{a}}$, You-Chen Chao ${ }^{\mathrm{b}}$, Lai-Fa Sheu $^{\mathrm{a}}$, Junn-Liang Chang ${ }^{\mathrm{c}}$, Shin Nieh ${ }^{\mathrm{a}}$ and \\ Jong-Shiaw Jin ${ }^{\mathrm{a}, *}$ \\ ${ }^{a}$ Department of Pathology, Tri-Service General Hospital, National Defense Medical Center, Taipei, Taiwan \\ ${ }^{\mathrm{b}}$ Division of Gastroenterology, Department of Internal Medicine, Tri-Service General Hospital, National Defense \\ Medical Center, Tapei, Taiwan \\ ${ }^{\mathrm{c}}$ Department of Clinical Pathology and Laboratory Medicine, Taoyuan Armed Forces General Hospital, Lungtan \\ Taoyuan County, Taiwan
}

\begin{abstract}
Objective: Fascin-1 is an actin-binding protein that promotes cell proliferation, adhesion and motility. We tested the hypothesis that fascin- 1 expression correlates with clinicopathological parameters of colorectal adenocarcinomas.

Methods: Immunohistochemical analysis of fascin-1 was performed in tissue microarrays of 91 surgical specimens, including 32 well, 33 moderately, and 26 poorly differentiated colorectal adenocarcinomas; and in 22 specimens from colorectal adenomas with dysplasia.

Results: Scattered fascin-1 expression was demonstrated in 9 control specimens of normal colonic glandular epithelia. Higher fascin-1 immunostaining scores were significantly associated with advanced dysplasia in colorectal adenomas (mild 4.2 \pm 1.3 , moderate $13.5 \pm 5.3$, and severe $22.5 \pm 6.7$ ) and high-grade histopathological differentiation of colorectal adenocarcinomas (grade I $88.6 \pm 9$, grade II $101 \pm 11$, and grade III $144 \pm 13$ ). Higher immunostaining scores of fascin-1 were also significantly associated with advanced T stage (T1: $42 \pm 10, \mathrm{~T} 2: 60 \pm 12, \mathrm{~T} 3: 108 \pm 12$, and T4: $142 \pm 15$ ). Higher fascin-1 scores were related with more advanced $\mathrm{M}$ and $\mathrm{N}$ stages of colorectal carcinomas, but not significant correlation.

Conclusions: Higher expression of fascin-1 correlates significantly with tumor grades and TNM stages in colorectal adenocarcinomas and also with levels of dysplastic change in colorectal adenomas.
\end{abstract}

Keywords: Colorectal cancer, colorectal adenoma, fascin-1, immunostaining scores, tissue microarray

\section{Introduction}

The fraction of colorectal adenocarcinoma is the most common histological type of total colon cancer,

\footnotetext{
*Corresponding author: Jong-Shiaw Jin, M.D., Ph.D., Department of Pathology, Tri-Service General Hospital, National Defense Medical Center, No. 325, Sec. 2, Cheng-Kung Road, Neihu 114, Taipei, Taiwan. Tel.: +886 2 87927155; Fax: +886 2 26913324; E-mail: jsjin@ndmctsgh.edu.tw.
}

accounting for $8.5 \%$ of all new malignancies [1]. Multiple factors, such as histopathological differentiation, depth of tumor invasion, and lymph node metastasis have been proven to play important roles in tumor prognosis [2-5]. Several studies demonstrated that both integrin and cadherin superfamilies contribute to maintaining cellular polarity and controlling epithelial differentiation in colonic epithelium [6,7]. Identification of mechanisms promoting tumor cell invasion may help direct creation of new therapies that can arrest 

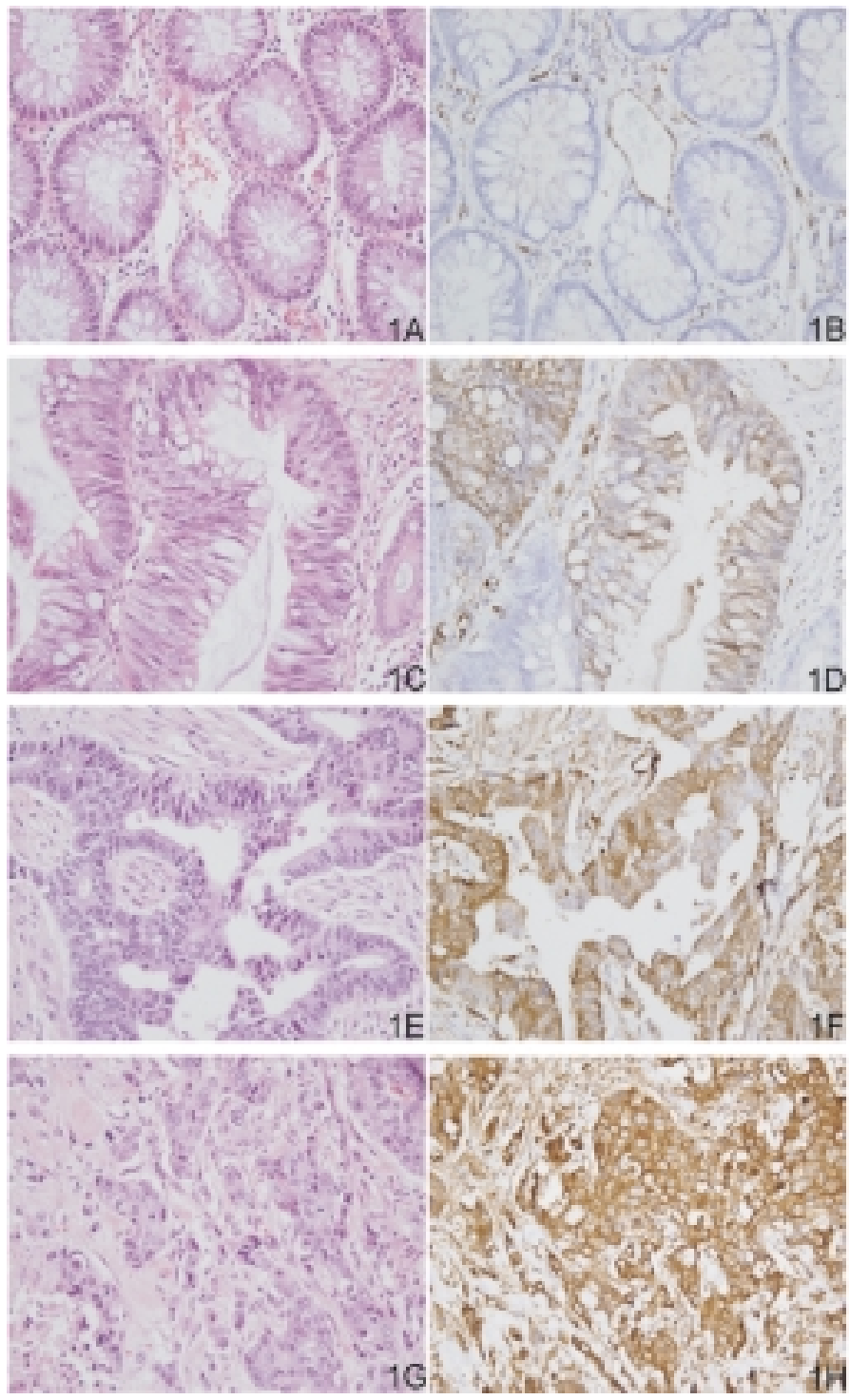

Fig. 1. Hematoxylin and eosin staining of non-neoplastic colon tissue (1A), well differentiated (1C), moderately differentiated (1E), and poorly differentiated $(1 \mathrm{G})$ colorectal adenocarcinomas; and immunohistochemical analysis of fascin-1 in non-neoplastic colon tissue (1B), well differentiated (1D), moderately differentiated (1F), and poorly differentiated (1H) colorectal adenocarcinomas. Original magnification X 400 .

local invasion and metastatic spread of disease. Recent cell culture study of colorectal adenocarcinoma showed fascin-1 over-expression was related to tumor invasiveness, proliferation, and differentiation [8].

Fascins are actin-binding proteins that induce cell membrane protrusion and motility [9]. In human body, the genomes encode 3 subtypes of the fascin family, including fascin-1, fascin-2, and fascin-3 [10-12]. Fascin-1 subtype is expressed widely in smooth muscle tissue, vascular endothelium, fibroblasts, lymphoid dendritic cells and neural crest cells $[8,13]$. However, fascin-2 expression is restricted to retinal photorecep- tor cells, and fascin-3 only appears in the testis [11, 12]. Fascin- 1 has been studied in different types of tumors, such as breast, colon, brain, esophagus, stomach, lung, urinary bladder, and even hematological malignancies [14-20]. However, the relationship between fascin-1 expression and clinicopathological parameters of colorectal adenocarcinomas is still vague.

In this study, we tested the hypothesis that higher expression of fascin-1 has prognostic significance in colorectal adenocarcinoma and malignant transformation in colorectal adenomas. Our results demonstrate that significantly increased fascin-1 immunostaining scores 
were not only associated with more advanced stages and poor survival in colorectal adenocarcinoma cases, but also higher degrees of dysplasia in colorectal adenoma.

\section{Materials and methods}

Paraffin-embedded tumor tissues were obtained and tissue microarray slides were constructed. We selected 22 colorectal adenoma cases, including 5 with mild dysplasia, 7 with moderate dysplasia, and 10 with severe dysplasia; and 91 cases of primary colorectal adenocarcinoma, including 32 well differentiated (glandular structure $>95 \%$ ), 33 moderately differentiated (glandular structure between $50 \%-95 \%$ ), and 26 poorly differentiated adenocarcinomas (glandular structure $<50 \%$ ). The histopathological differentiation of colorectal adenocarcinoma was determined according to the WHO classification criteria for tumors [2].

One core was taken from a selected area of each paraffin-embedded tumor tissue and tissue microarray slides were constructed according to a previously published method [21]. Each representative core in the tissue microarray slide was $1.5 \mathrm{~mm}$ in diameter. The tissue microarray slide showed uniform H\&E staining as did the original paraffin-embedded specimens. Each pathological diagnosis in these cases was reviewed by at least two experienced pathologists who were blinded to each other's result. If the discrepancy developed, the third opinion of another pathologist was consulted. All tumors were pathologically staged according to the 1997 American Joint Committee on Cancer (AJCC/TNM system). Normal colonic tissues were obtained from 9 cases and were taken $4 \mathrm{~cm}$ from the neoplasm. None of these cases had ever received radiation or chemotherapy before surgery.

\subsection{Immunohistochemistry}

Tissue microarray sections were de-waxed in xylene, rehydrated in alcohol, and immersed in 3\% hydrogen peroxide for 5 minutes to suppress endogenous peroxidase activity. Antigen retrieval was performed by heating $\left(100^{\circ} \mathrm{C}\right)$ each section for 30 minutes in $0.01 \mathrm{~mol} / \mathrm{L}$ sodium citrate buffer $(\mathrm{pH} 6.0)$. After 3 rinses (each for 5 minutes in phosphate buffered saline $[\mathrm{PBS}]$ ), sections were incubated for 1 hour at room temperature with a monoclonal mouse anti-human fascin-1 antibody (1:100, NeoMarkers, Fremont, USA) diluted in PBS as previous study [22,23]. After 3 washes (each for 5 minutes in PBS), sections were incubated with biotin-labeled secondary immunoglobulin (1:100, DAKO, Glostrup, Denmark) for 1 hour at room temperature. After 3 additional washes, peroxidase activity was developed with DAB (DAKO, Glostrup, Denmark) at room temperature. Sections of non-neoplastic muscle tissues (known to stain positive for fascin-1) were used as a positive control and normal columnar epithelia of breast were used as a negative control [24].

For evaluation of immunoreactivity and histological appearance, all tissue microarray experiments were repeated 3 times and the slides were examined and scored by two authors concurrently. These replicated results of each author were averaged. For assessment of fascin1 immunostaining scores, the intensity of cytoplasmic and plasma membrane immunostaining was scored on a scale of 0 (no staining) to 4 (strongest intensity), and the percentage of cells with stained cytoplasm or plasma membrane was estimated at each intensity. The percentage of cells (from 0 to 100) was multiplied by the corresponding immunostaining intensity (from 0 to 4) to obtain immunostaining scores ranging from 0 to 400 .

\subsection{Statistical analysis}

All results are expressed as mean \pm standard error of the mean (S.E.M.). The standard error of the mean (S.E.M) was analyzed on these various cases of the same clinical stage and histological grade. The immunostaining scores of fascin-1 in colorectal adenomas and adenocarcinomas were compared with the score in normal colonic epithelia. Statistical analysis was performed using the Student $t$-test between groups and a p-value of less than 0.05 was considered to be statistically significant. SigmaState software (Jandel Scientific, San Rafael, CA, USA) was used to perform linear regression testing to analyze the relationship between fascin-1 expression and clinicopathological parameters.

\section{Results}

\subsection{Clinicopathological characteristics}

Among all 91 colorectal adenocarcinoma cases in the study, there were 51 males and 40 females. The age distribution ranged from 47 to 75 years with a mean of 65.5. Other information recorded included histopathological differentiation, tumor classification, and staging distributions, which are listed as Table 1. 


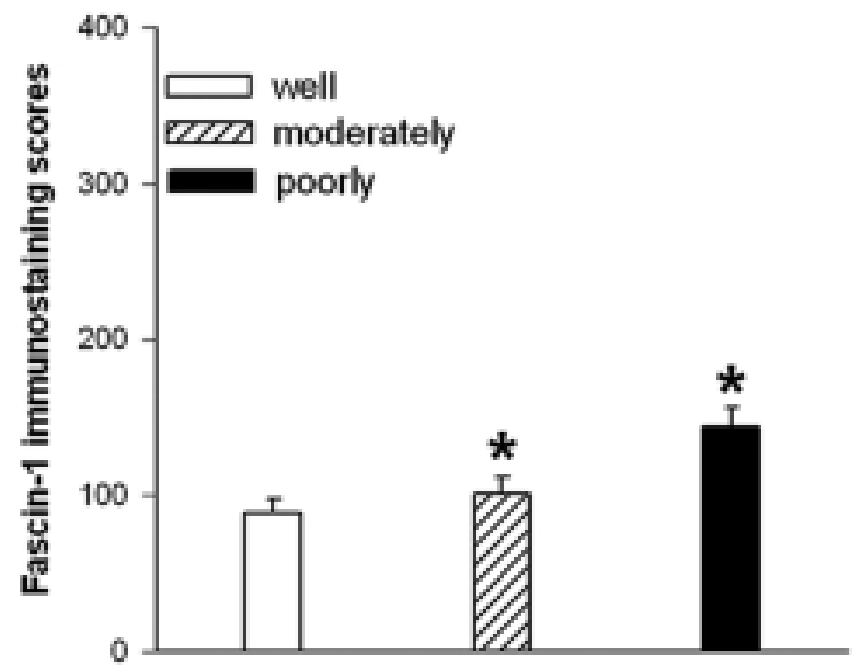

Fig. 2. Immunostaining scores of fascin-1 in well differentiated, moderately differentiated, and poorly differentiated colorectal adenocarcinoma. *Indicates statistical significance in comparison to well differentiated colorectal adenocarcinoma $(p<0.05)$.

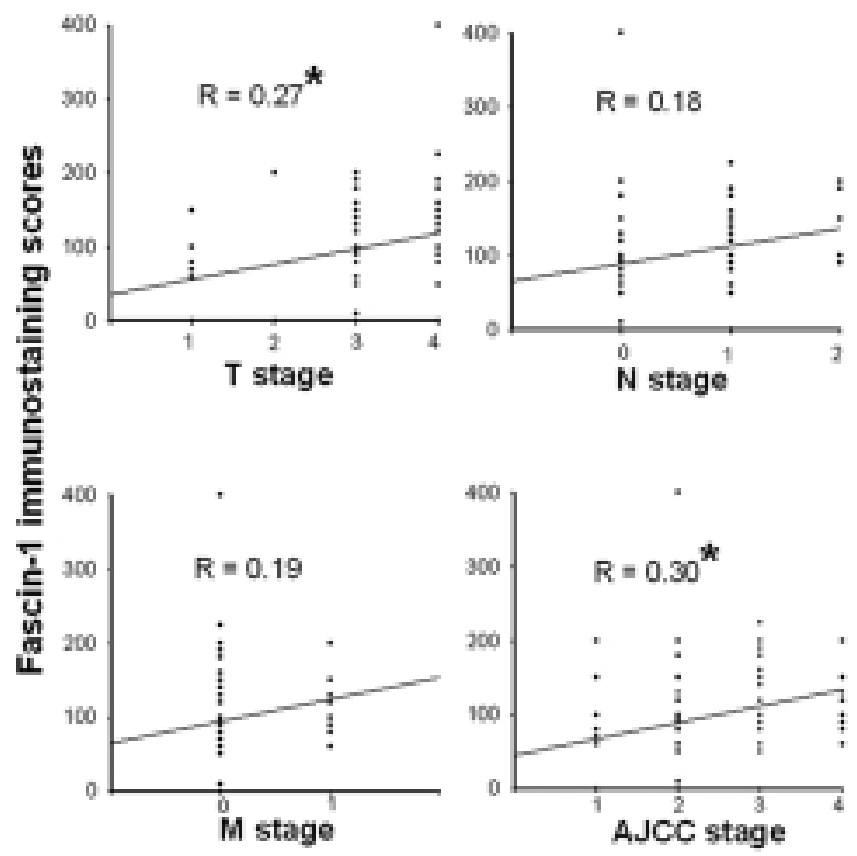

Fig. 3. Clinicopathological correlations with fascin-1 immunostaining scores in colorectal adenocarcinoma. *Indicates statistical significance of linear regression testing $(p<0.05)$.

\subsection{Fascin-1 expression in colorectal adenocarcinoma}

The immunostaining results of fascin-1 expression in colorectal adenocarcinoma are summarized in Fig. 1 and Table 1. Non-neoplastic colonic glands (Fig. 1B) revealed only scattered expression of fascin- 1 and the average immunostaining score was only $0.5 \pm 0.3$.
However, fascin-1 immunoreactivity was seen on the cell membrane and cytoplasm in most colorectal adenocarcinoma cases. The fascin-1 immunostaining scores in well differentiated (Fig. 1D, $88.6 \pm 9.5$ ), moderately differentiated (Fig. 1F, $101.2 \pm 10.9$ ), and poorly differentiated (Fig. 1H, $143.5 \pm 12.6$ ) colorectal adenocarcinomas were significantly higher than in normal colonic tissue. Otherwise, the fascin-1 scores in mod- 

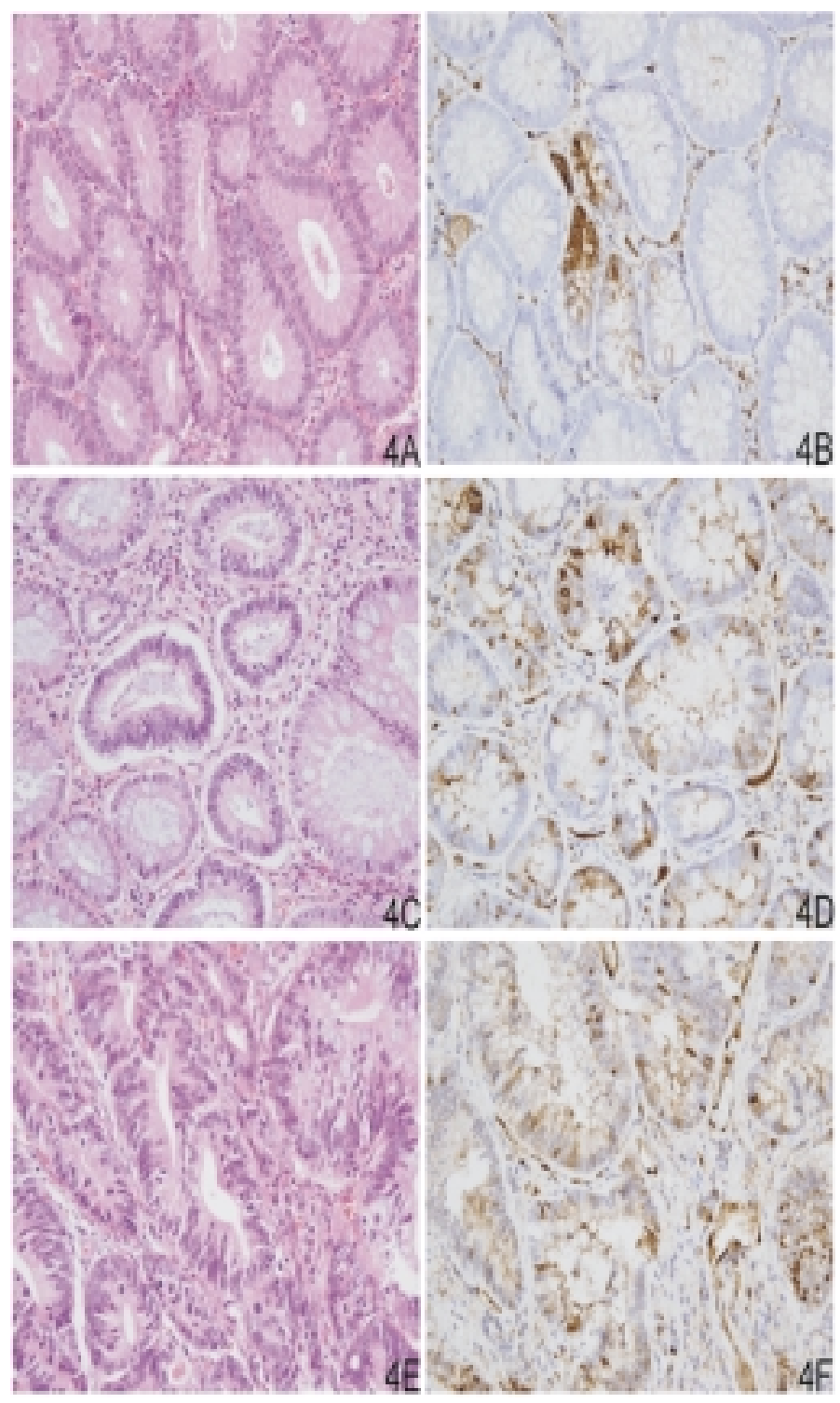

Fig. 4. Hematoxylin and eosin staining of mild dysplastic (4A), moderate dysplastic (4C), and severe dysplastic (4E) colorectal adenomas; and immunohistochemical analysis of fascin-1 in mild dysplastic (4B), moderate dysplastic (4D), and severe dysplastic (4F) colorectal adenomas. Original magnification X 400 .

erately and poorly differentiated colorectal adenocarcinoma were significantly higher than in well differentiated ones (Fig. 2).

\subsection{The expressions of fascin-1 correlate with clinical stages}

Linear regression testing was performed to analyze the relationship between fascin-1 immunostaining scores and clinical TNM stages. The average immunostaining scores of fascin- 1 were $42.2 \pm 10.3$ for stage
$\mathrm{T} 1,60.0 \pm 11.5$ for stage $\mathrm{T} 2,107.7 \pm 12.2$ for stage $\mathrm{T} 3$, and $141.8 \pm 15.3$ for stage T4 cases of colorectal adenocarcinomas. Advanced $\mathrm{T}$ stage correlated significantly with higher fascin- 1 immunostaining scores $(P<0.05)$. However, the more advanced $\mathrm{M}$ or $\mathrm{N}$ stages of colorectal adenocarcinoma cases did not significantly correlate with higher intensities, greater percentages of tumor staining and immunostaining scores of fascin-1 expression. In addition, the immunostaining scores of various clinical stages of fascin- 1 cases were listed as follows: $45.2 \pm 7.5$ for stage I, $82.6 \pm 13.3$ 
Table 1

The immunostaining patterns of fascin-1 and clinicopatholigcal parameters of colorectal adenocarcinomas and non-neoplastic colonic tissues

\begin{tabular}{|c|c|c|c|c|c|}
\hline & $\begin{array}{l}\text { No. of } \\
\text { cases }\end{array}$ & $\begin{array}{l}\text { Average } \\
\text { intensity }\end{array}$ & $\begin{array}{l}\text { Average } \\
\% \text { tumor }\end{array}$ & $\begin{array}{l}\text { Average } \\
\text { score }\end{array}$ & Correlation \\
\hline \multicolumn{6}{|l|}{ Histological grading } \\
\hline Normal colon tissue & 9 & $0.3 \pm 0.1$ & $1.5 \pm 0.7$ & $0.5 \pm 0.3$ & \multirow{4}{*}{$\begin{array}{c}\text { Positive } \\
\text { correlation } \\
(P<0.05)\end{array}$} \\
\hline Well differentiated & 32 & $1.3 \pm 0.2$ & $58.4 \pm 6.8$ & $88.6 \pm 9.5$ & \\
\hline Moderately differentiated & 33 & $1.5 \pm 0.2$ & $63.5 \pm 6.1$ & $101.2 \pm 10.9$ & \\
\hline Poorly differentiated & 26 & $2.1 \pm 0.3$ & $68.2 \pm 6.5$ & $143.5 \pm 12.6$ & \\
\hline \multicolumn{6}{|l|}{ TNM stage } \\
\hline \multicolumn{6}{|l|}{ T stage } \\
\hline $\mathrm{T} 1$ & 10 & $0.6 \pm 0.1$ & $33.9 \pm 5.9$ & $42.2 \pm 10.3$ & \multirow{4}{*}{$\begin{array}{c}\text { Positive } \\
\text { correlation } \\
(P<0.01)\end{array}$} \\
\hline $\mathrm{T} 2$ & 4 & $1.3 \pm 0.2$ & $47.5 \pm 2.5$ & $60.0 \pm 11.5$ & \\
\hline $\mathrm{T} 3$ & 48 & $1.6 \pm 0.2$ & $61.7 \pm 6.3$ & $107.7 \pm 12.2$ & \\
\hline $\mathrm{T} 4$ & 29 & $2.1 \pm 0.3$ & $67.8 \pm 5.2$ & $141.8 \pm 15.3$ & \\
\hline \multicolumn{6}{|l|}{$\mathrm{N}$ stage } \\
\hline NO & 58 & $1.4 \pm 0.1$ & $53.6 \pm 5.4$ & $93.1 \pm 11.8$ & \multirow{3}{*}{$\begin{array}{c}\text { No } \\
\text { correlation } \\
(P=0.07)\end{array}$} \\
\hline N1 & 26 & $1.7 \pm 0.2$ & $63.7 \pm 4.1$ & $113.3 \pm 19.1$ & \\
\hline $\mathrm{N} 2$ & 7 & $2.3 \pm 0.4$ & $70.1 \pm 5.3$ & $150.9 \pm 27.4$ & \\
\hline \multicolumn{6}{|l|}{ M stage } \\
\hline M0 & 78 & $1.4 \pm 0.2$ & $66.8 \pm 4.4$ & $132.9 \pm 11.0$ & \multirow{2}{*}{$\begin{array}{l}\text { No correlation } \\
(P=0.11)\end{array}$} \\
\hline M1 & 13 & $2.2 \pm 0.3$ & $69.4 \pm 5.6$ & $150.2 \pm 19.9$ & \\
\hline \multicolumn{6}{|l|}{ Clinical stage } \\
\hline Stage I & 13 & $0.8 \pm 0.1$ & $36.2 \pm 5.5$ & $45.2 \pm 7.5$ & \multirow{4}{*}{$\begin{array}{c}\text { Positive } \\
\text { correlation } \\
(P<0.05)\end{array}$} \\
\hline Stage II & 39 & $1.4 \pm 0.2$ & $52.5 \pm 6.6$ & $82.6 \pm 13.3$ & \\
\hline Stage III & 26 & $1.8 \pm 0.3$ & $65.4 \pm 4.5$ & $132.8 \pm 15.7$ & \\
\hline Stage IV & 13 & $2.2 \pm 0.3$ & $69.4 \pm 5.6$ & $150.2 \pm 19.9$ & \\
\hline
\end{tabular}

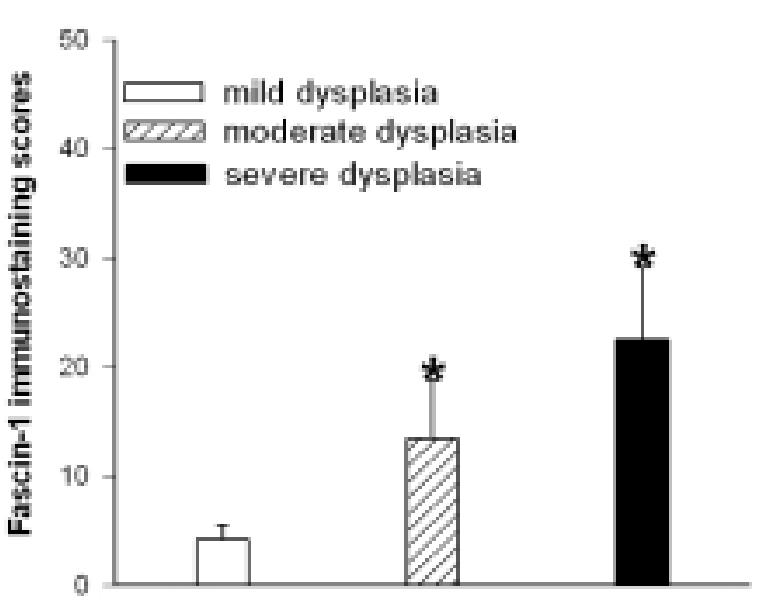

Fig. 5. Immunostaining scores of fascin-1 in mild dysplastic, moderate dysplastic, and severe dysplastic colorectal adenomas. *Indicates statistical significance in comparison to mild dysplastic colorectal adenomas $(p<0.05)$.

for stage II, $132.8 \pm 15.7$ for stage III, and 150.2 \pm 19.9 for stage IV. Higher immunostaining scores of fascin1 also correlated significantly with advanced clinical stages $(P<0.05$, Fig. 3 and Table 1$)$.

\subsection{Fascin-1 expression in colorectal adenoma}

The immunostaining results of fascin- 1 expression in colorectal adenomas with various degrees of dyspla- sia are summarized in Fig. 4 and Table 2. The fascin1 immunostaining scores in colorectal adenomas with mild dysplasia (Fig. 4B, $4.2 \pm 1.3$ ), moderate dysplasia (Fig. 4D, $13.5 \pm 5.3$ ), and severe dysplasia (Fig. $4 \mathrm{~F}, 22.5 \pm 6.7$ ) were significantly higher than in normal colonic tissue, but lower than in colorectal adenocarcinomas. Otherwise, the fascin-1 scores in moderate and severe dysplasia of colorectal adenomas were significantly higher than in mild dysplasia (Fig. 5).

\section{Discussion}

In the current study, we demonstrated that fascin1 immunostaining scores indeed correlate with tumor progression and aggressiveness of colorectal adenocarcinomas. In addition, fascin-1 is a satisfactory biomarker to predict the malignant transformation of colorectal columnar epithelia. This marker may be valuable in helping the pathologist discriminate between benign colonic glandular epithelia and malignant colorectal adenocarcinomas, especially in small lesions with good differentiation.

Multiple factors (such as familial inherited disease, genetic mutation, cellular cycle regulator dysfunction, and imbalances in growth factors) increase the incidence and progression of colorectal cancer [2]. In a 
Table 2

Immunostaining scores of fascin- 1 in 22 colorectal adenoma cases

\begin{tabular}{lccrrc}
\hline $\begin{array}{l}\text { Degree of } \\
\text { dysplasia }\end{array}$ & $\begin{array}{c}\text { No. of } \\
\text { cases }\end{array}$ & Intensity & \% staining & Total score & T-test* \\
\hline Mild & 5 & $0.6 \pm 0.2$ & $5.3 \pm 0.9$ & $4.2 \pm 1.3$ & Significant \\
Moderate & 7 & $0.7 \pm 0.3$ & $15.0 \pm 4.6$ & $13.5 \pm 5.3$ & difference \\
Severe & 10 & $1.1 \pm 0.2$ & $16.5 \pm 5.5$ & $22.5 \pm 6.7$ & $(P<0.05)$ \\
\hline
\end{tabular}

*Indicates statistical significance in comparison with normal colorectal glan-

dular tissue $(p<0.05)$.

recent cell-culture study, intercellular and cell-matrix adhesion molecules were shown to play important roles in regulating cell polarity, differentiation, proliferation, migration and invasion in colorectal cancer [23]. Upregulation of fascin in tumor cells may be associated with defects in cell adhesion and may increase the risk of tumor progression [8]. However, further studies are needed to investigate the mechanisms between fascin1 expression and carcinogenesis in colorectal carcinomas [25].

In our study, all tumor tissues were placed in a single tissue-array slide. The tissue microarray technique is a powerful tool for simultaneous histological and immunohistochemical evaluation of tumors [26]. Previous studies measuring immunohistochemical intensity of individual cases were limited because of the variability of the chemical signal generated under different environmental conditions [26]. Recent results support the reliability of immunohistochemistry conducted on tissue microarray slides [26]. In our study, the clear cut difference in fascin staining between colonic adenocarcinoma tissue and normal glandular epithelia validated the use of tissue microarray slides.

Fascin-1, a 55-kDa globular protein, causes the aggregation of $\mathrm{F}$ actin into parallel bundles, which rearranges the cytoskeleton and promotes cellular motility $[9,27]$. The gene encoding fascin-1 in humans is located at chromosome 7q22 [24]. In normal epithelia of the biliary duct, breast, colon, ovary, pancreas and stomach, the expression of fascin-1 is often scattered or completely negative [24]. In contrast, higher expression of fascin-1 in lung, gastric, esophageal and breast carcinomas had been shown to correlate with poor prognosis and/or to decrease survival time [17, 18,28-30]. However, in colorectal cancer, the utilization of fascin-1 over-expression as a molecular marker to predict the clinical outcome is still lacking [25]. Our results clearly demonstrated that the expression of fascin-1 is effective in predicting tumor behavior, such as tumor progression, invasion, and malignant transformation of colorectal epithelial-derived tumors.

In conclusion, higher fascin-1 scores were correlated significantly with more advanced TNM stages of col- orectal carcinomas. Although there are still unknown mechanisms in tumor progression, we demonstrated that fascin-1 is an applicable biomarker to predict clinicopathological parameters. Therefore, the development of effective pharmacological agents to target the fascin-1 pathway may prolong survival time and slow tumor progression in patients with colorectal adenocarcinomas.

\section{Acknowledgments}

This study was supported by grants from National Science Counsel, NSC94-2320-B-016-017, and TriService General Hospital, TSGH-C95-16-S05, Taiwan, R. O. C.

\section{References}

[1] J.D. Potter, Colorectal cancer: molecules and population, $J$ Natl Cancer Inst 91 (1999), 916-932.

[2] S.R. Hemilton and L.A. Aaltonen, WHO classification of tumors: tumors of the digestive system, Lyon: IRAC press, 2000, 105-119.

[3] S. Sasaki, T. Masaki, N. Umetani et al., Characteristics in primary signet-ring cell carcinoma of the colorectum, from clinicopathological observation, Jpn J Clin Oncol 28 (1998), 202-206.

[4] B. Cagir, M.W. Nagy, A. Topham et al., Adenosquamous carcinoma of the colon, rectum, and anus: epidemiology, distribution, and survival characteristics, Dis Colon Rectum 42 (1999), 258-263.

[5] J.P. Cerottini, S. Caplin, S. Pampallona et al., Prognostic factors in colorectal cancer, Oncol Rep 6 (1999), 409-414.

[6] M. Pignatelli and W.F. Bodmer, Integrin cell adhesion molecules and colorectal cancer, J Pathol 162 (1990), 95-97.

[7] M. Pignatelli, D. Liu, M.M. Nasim et al., Morphoregulatory activities of E-cadherin and beta-1 integrins in colorectal tumour cells, Br J Cancer 66 (1992), 629-634.

[8] A.U. Jawhari, A. Buda, M. Jenkins et al., Fascin, an actinbundling protein, modulates colonic epithelial cell invasiveness and differentiation in vitro, Am J Pathol 162 (2003), 6980.

[9] J.C. Adams, Roles of fascin in cell adhesion and motility, Curr Opin Cell Biol 16 (2004), 590-596. 
[10] F.M. Duh, F. Latif, Y. Weng et al., cDNA cloning and expression of the human homolog of the sea urchin fascin and Drosophila singed genes which encodes an actin-bundling protein, DNA Cell Biol 13 (1994), 821-827.

[11] B.E. Tubb, S. Bardien-Kruger, C.D. Kashork et al., Characterization of human retinal fascin gene (FSCN2) at $17 \mathrm{q} 25$ : close physical linkage of fascin and cytoplasmic actin genes, Genomics 65 (2000), 146-156.

[12] B. Tubb, D.J. Mulholland, W. Vogl et al., Testis fascin (FSCN3): a novel paralog of the actin-bundling protein fascin expressed specifically in the elongate spermatid head, Exp Cell Res 275 (2002), 92-109.

[13] G. Mosialos, M. Birkenbach, S. Ayehunie et al., Circulating human dendritic cells differentially express high levels of a 55-kd actin-bundling protein, Am J Pathol 148 (1996), 593600.

[14] A. Grothey, R. Hashizume, A.A. Sahin et al., Fascin, an actinbundling protein associated with cell motility, is upregulated in hormone receptor negative breast cancer, $\mathrm{Br} J$ Cancer $\mathbf{8 3}$ (2000), 870-873.

[15] A.A. Roma and R.A. Prayson, Fascin expression in 90 patients with glioblastoma multiforme, Ann Diagn Pathol 9 (2005), 307-311.

[16] J.J. Xie, L.Y. Xu, H.H. Zhang et al., Role of fascin in the proliferation and invasiveness of esophageal carcinoma cells, Biochem Biophys Res Commun 337 (2005), 355-362.

[17] Y. Hashimoto, Y. Shimada, J. Kawamura et al., The prognostic relevance of fascin expression in human gastric carcinoma, Oncology 67 (2004), 262-270.

[18] G. Pelosi, U. Pastorino, F. Pasini et al., Independent prognostic value of fascin immunoreactivity in stage I nonsmall cell lung cancer, Br J Cancer 88 (2003), 537-547.

[19] G.X. Tong, H. Yee, L. Chiriboga et al., Fascin-1 expression in papillary and invasive urothelial carcinomas of the urinary bladder, Hum Pathol 36 (2005), 741-746.

[20] G. Fan, P. Kotylo, R.S. Neiman et al., Comparison of fascin expression in anaplastic large cell lymphoma and Hodgkin disease, Am J Clin Pathol 119 (2003), 199-204.

[21] J. Jacquemier, C. Ginestier, F. Bertucci, J. Geneix and D. Birnbaum, Tissue microarrays: a powerful tool in transfer and quality control in oncology, Bull Cancer 90 (2003), 31-38.

[22] W.C. Tsai, L.F. Sheu, S. Nieh et al., Association of EMMPRIN and fascin expression in renal cell carcinoma: correlation with clinicopathological parameters, World J Urol (2006), Article in press.

[23] J.S. Jin, C.P. Yu, G.H. Sun et al., Increasing expression of fascin in renal cell carcinoma associated with clinicopathological parameters of aggressiveness, Histol Histopathol 21 (2006), 1287-1293.

[24] Y. Hashimoto, M. Skacel and J.C. Adams, Roles of fascin in human carcinoma motility and signaling: prospects for a novel biomarker? Int J Biochem Cell Biol 37 (2005), 1787-1804.

[25] A. Buda and M. Pignatelli, Cytoskeletal network in colon cancer: from genes to clinical application, Int J Biochem Cell Biol 36 (2004), 759-765.

[26] J.S. Lam, A.S. Belldegrum and R.A. Figlin, Tissue arraybased predictions of pathobiology, prognosis and response to treatment for renal cell carcinoma therapy, Clin Cancer Res 10 (2004), 6304s-6309s.

[27] N. Kureishy, V. Sapountzi, S. Prag et al., Fascins, and their roles in cell structure and function, Bioessays 24 (2002), 350361.

[28] J.C. Adams, Fascin protrusions in cell interactions, Trends Cardiovasc Med 14 (2004), 221-226.

[29] Y. Hashimoto, T. Ito, H. Inoue et al., Prognostic significance of fascin overexpression in human esophageal squamous cell carcinoma, Clin Cancer Res 11 (2005), 2597-2605.

[30] B.J. Yoder, E. Tso, M. Skacel et al., The expression of fascin, an actin-bundling motility protein, correlates with hormone receptor-negative breast cancer and a more aggressive clinical course, Clin Cancer Res 11 (2005), 186-192. 


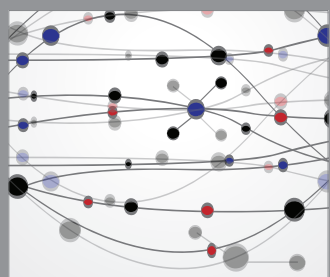

The Scientific World Journal
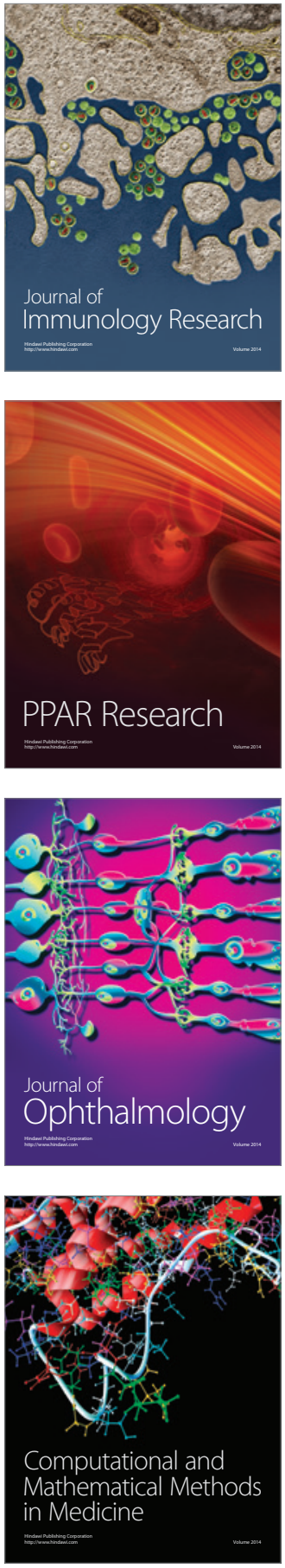

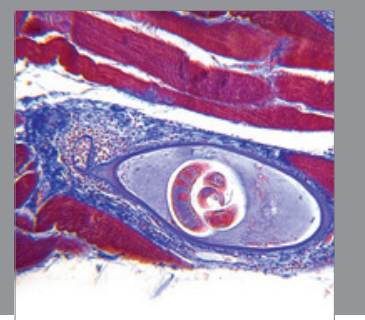

Gastroenterology

Research and Practice
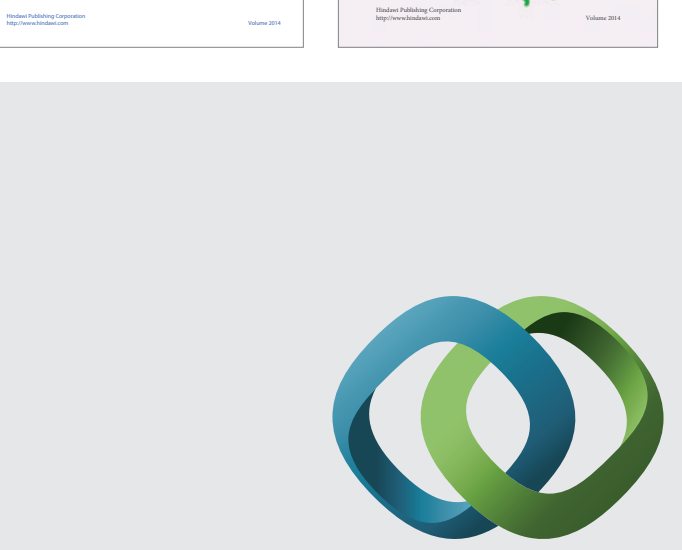

\section{Hindawi}

Submit your manuscripts at

http://www.hindawi.com
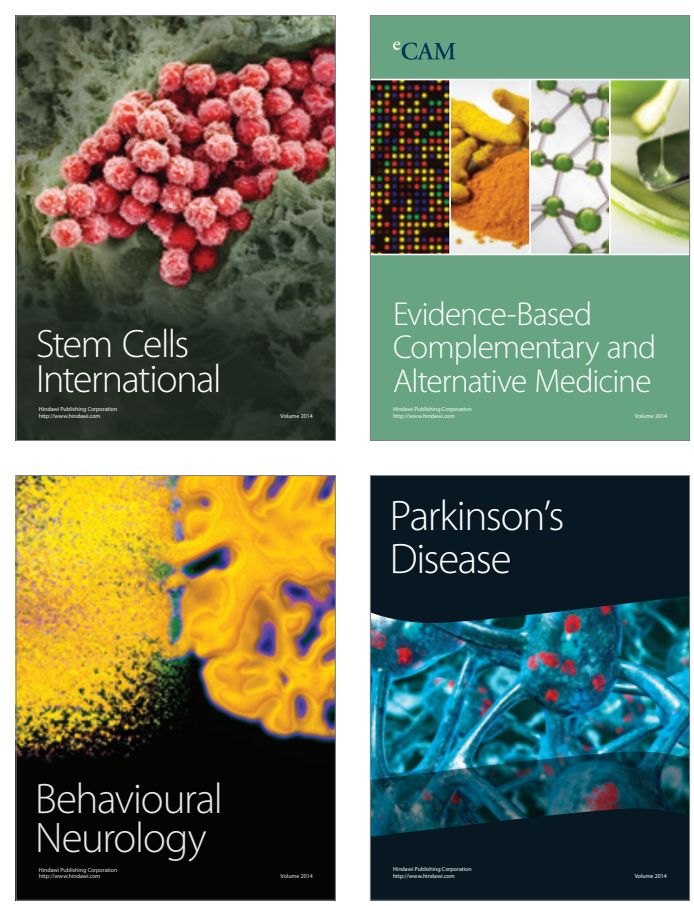

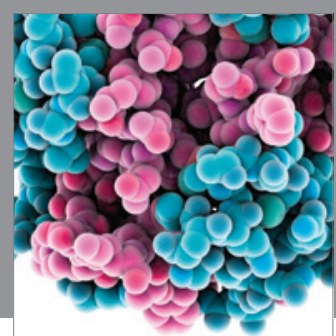

Journal of
Diabetes Research

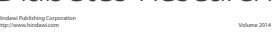

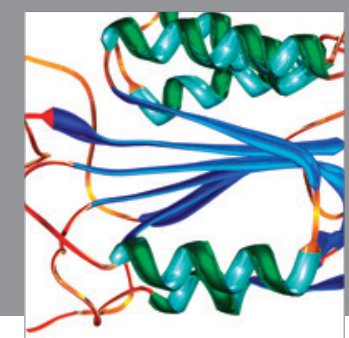

Disease Markers
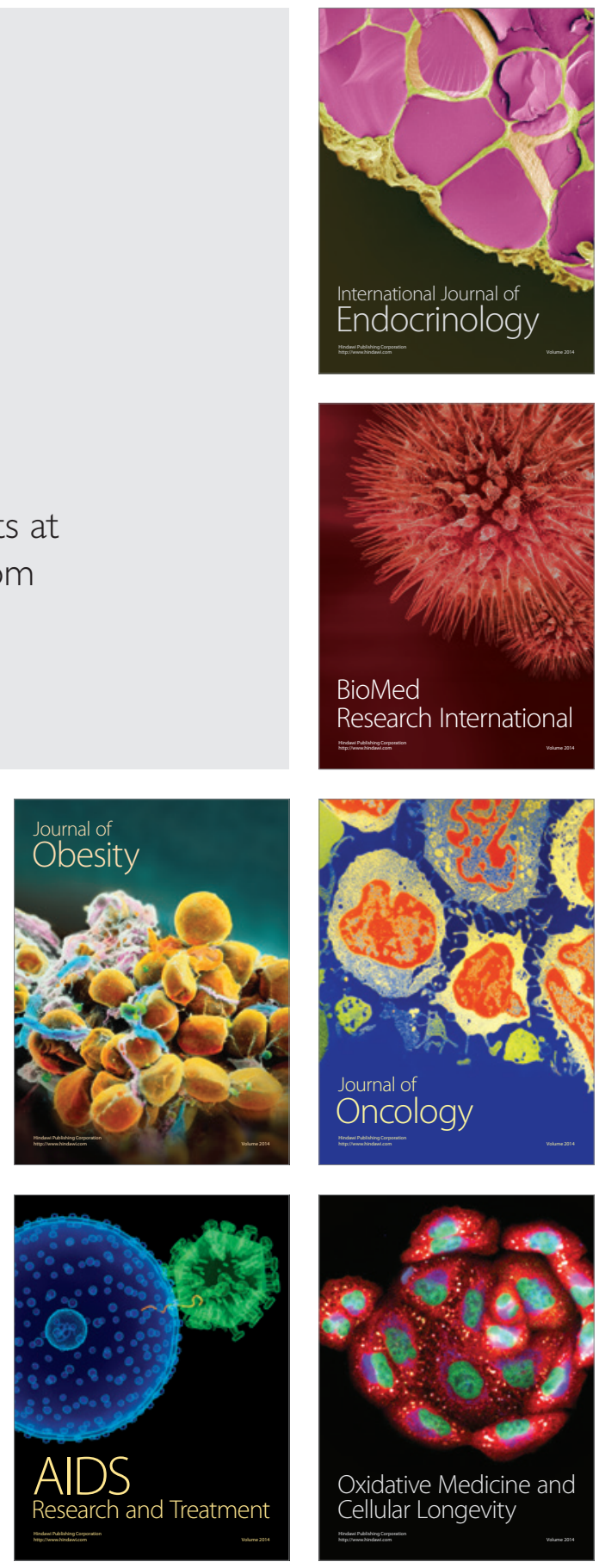